\section{Cureus}

\title{
Single Session Robotic Radiosurgery for a Patient with Low Risk Prostate Cancer
}

\author{
Alexander Muacevic ${ }^{1}$, Christoph Fuerweger ${ }^{2}$, B. Wowra ${ }^{3}$, Michael Staehler ${ }^{4}$ \\ 1. European CyberKnife Center Munich, Munich, DEU 2. Cyberknife, European CyberKnife Center \\ Munich 3. European Cyberknife Center Munich-Grosshadern 4. Department of Urology, University \\ Hospital, Ludwig Maximilian University of Munich, Munich, DEU
}

$\square$ Corresponding author: Alexander Muacevic, alexander.muacevic@cureus.com Disclosures can be found in Additional Information at the end of the article

\section{Abstract}

Here, we describe the first patient treated by single session robotic radiosurgery for low risk prostate cancer. The initial PSA of this 70 -year-old patient was $5.05 \mathrm{ng} / \mathrm{dl}$ and transrectal biopsy confirmed a Gleason score 3 $+3=6$ tumor. The patient refused a standard surgical and conventional radiation treatment. The combined choline PET/CT before treatment did not show any additional tumor locations and MRI excluded prostate capsule infiltration. Robotic radiosurgery was offered upon the patient's request as an individual treatment attempt. After ultrasound-guided transrectal fiducial implantation, a highly conformal treatment plan was generated and the patient was treated in April 2008 with 19 Gy to the $70 \%$ isodose in a single session. The treatment was tolerated very well with the minor dysuretic symptoms during the first two weeks after the procedure. The PSA continuously dropped in the following years and measured $0.07 \mathrm{ng} / \mathrm{dl}$ in July 2014. During this period, no significant clinical symptoms were detected, and the patient experienced no rectal or urinary toxicity. This is the first published patient treated with single session prostate radiosurgery, and we believe a prospective trial is warranted to investigate further the options of this attractive treatment concept for low-grade prostate cancer patients.

Categories: Radiation Oncology, Urology

Keywords: prostate cancer, cyberknife $@$, radiosurgery, single fraction

\section{Introduction}

The use of hypofractionated CyberKnife stereotactic radiotherapy as treatment for early-stage prostate cancer has been described recently [1-5]. Hypofractionated stereotactic treatment is suggested to reduce the volume of adjacent tissue receiving high dose radiation and to apply a much larger, ablative dose of radiation per treatment fraction. Prostate movement prevents the application of radiosurgery by conventional radiation systems as the prostate can move up to 1.5 in every direction during treatment delivery [6-7]. To compensate the effects of prostate movement, a larger radiotherapy planning target volume is usually used.

Robotic image-guided tumor tracking might overcome this technical limitation of conventional radiation therapy [8-9]. Furthermore, hypofractionated radiosurgical treatment may be advantageous for prostate cancer as contemporary radiobiological data suggest the $\alpha / \beta$ ratio for prostate cancer tissue may be as low as $1.5 \mathrm{~Gy}$ [10]. This value of $\alpha / \beta$ is comparable to, if not lower, than for late-responding normal tissues [10]. Assuming that $\alpha / \beta$ is that low and the underlying technology can achieve a precise targeting of the prostate by image-guided organ tracking, the ultimate goal for patient comfort would be to administer only a single fraction of 
radiation if safety and efficacy were warranted. This radical approach has not been employed so far as fear for unpredicted toxicity and lack of long-term local tumor control exists. Here, we present a six-year follow up after single session image-guided robotic radiosurgery for a patient with low risk prostate cancer.

\section{Case Presentation}

Informed consent was obtained before treatment, and the patient was clearly informed that the standard therapy in his condition would be either active surveillance, surgery, external radiation, or hormonal therapy. He was further told that radiosurgery is not considered standard therapy and the indication was set on an individual treatment approach based on his particular wish. Treatment was done in April 2008.

\section{Robotic radiosurgery}

The CyberKnife robotic radiosurgery system (Accuray Incorporated, Sunnyvale, CA, USA) has been described elsewhere [5]. Briefly, it consists of a 6-MV compact linear accelerator (LINAC) mounted on a computer-controlled six-axis robotic manipulator. Integral to the system are orthogonally positioned $\mathrm{x}$-ray cameras, which acquire images during treatment. The images are processed automatically to identify radiographic features and registered to the treatment planning study to measure the position of the treatment site in real time [10]. The system adapts to changes in target position during treatment by acquiring X-ray images repeatedly and then adjusting the direction of the treatment beam. In contrast to a gantry-mounted LINAC, the treatment beam can be directed at the target from nearly anywhere around the patient, limited only by obstacles such as the treatment couch.

\section{Preparation}

Four fiducial seeds were placed under transrectal ultrasound guidance, using the transrectal approach and a biplanar ultrasound probe, with local anesthesia. 


\section{Cureus}

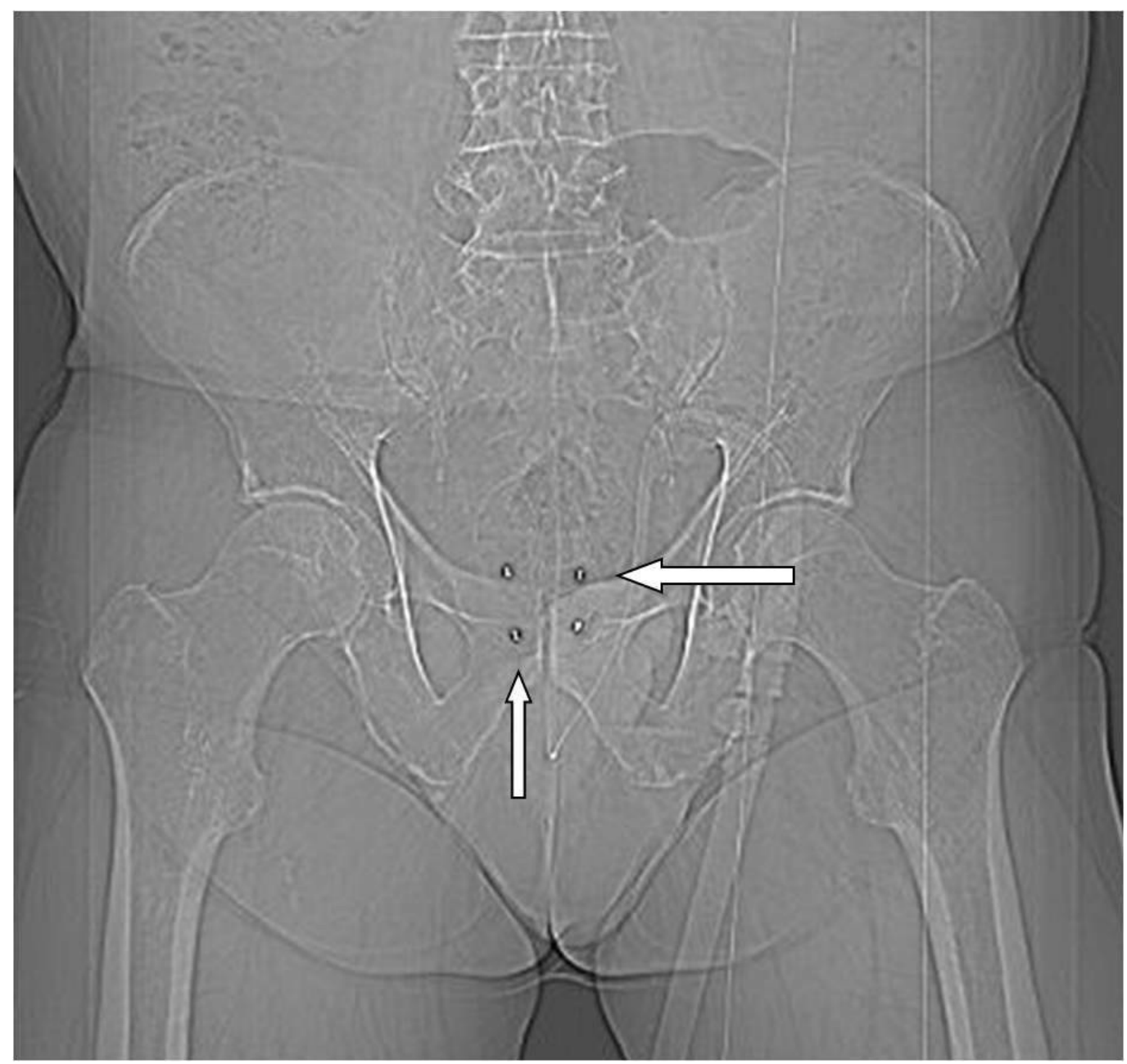

\section{FIGURE 1: Fiducial placement}

Arrows show an ideal placement of four gold fiducials (two upper part, two lower part) to the prostate for optimal tracking of translations and rotations during treatment.

Prophylactic antibiotics were given to reduce the risk of infection. Planning CT and MRI (to determine the anatomical borders of the prostate) studies were performed immediately after fiducial placement. No endorectal coil was allowed. CT, MRI, and choline PET/CT scans were performed for treatment planning. The planning CT and MRI scans were done with a Foley catheter in place to define the course of the urethra through the CTV, to potentially give more stabilization to the prostate and to allow the bladder to empty during the course of treatment. No other bowel/urinary preparation procedures were performed.

\section{Treatment planning}

Treatment planning started immediately after CT and MRI imaging. A radial margin of $2 \mathrm{~mm}$ was added around the prostate to create the planning target volume. Proximal seminal vesicle coverage was not added. The posterior PTV margin was reduced to zero. The patient received a minimal dose of $19 \mathrm{~Gy}$ to the $70 \%$ isodose enclosing the PTV. The urethra (prostatic part only) received a maximum dose of $20.4 \mathrm{~Gy}$, the mean dose was $17.4 \mathrm{~Gy}$. The rectum received a maximum dose of $16.7 \mathrm{~Gy}$, the V10 Gy was 4.2 cc. 


\section{Cureus}

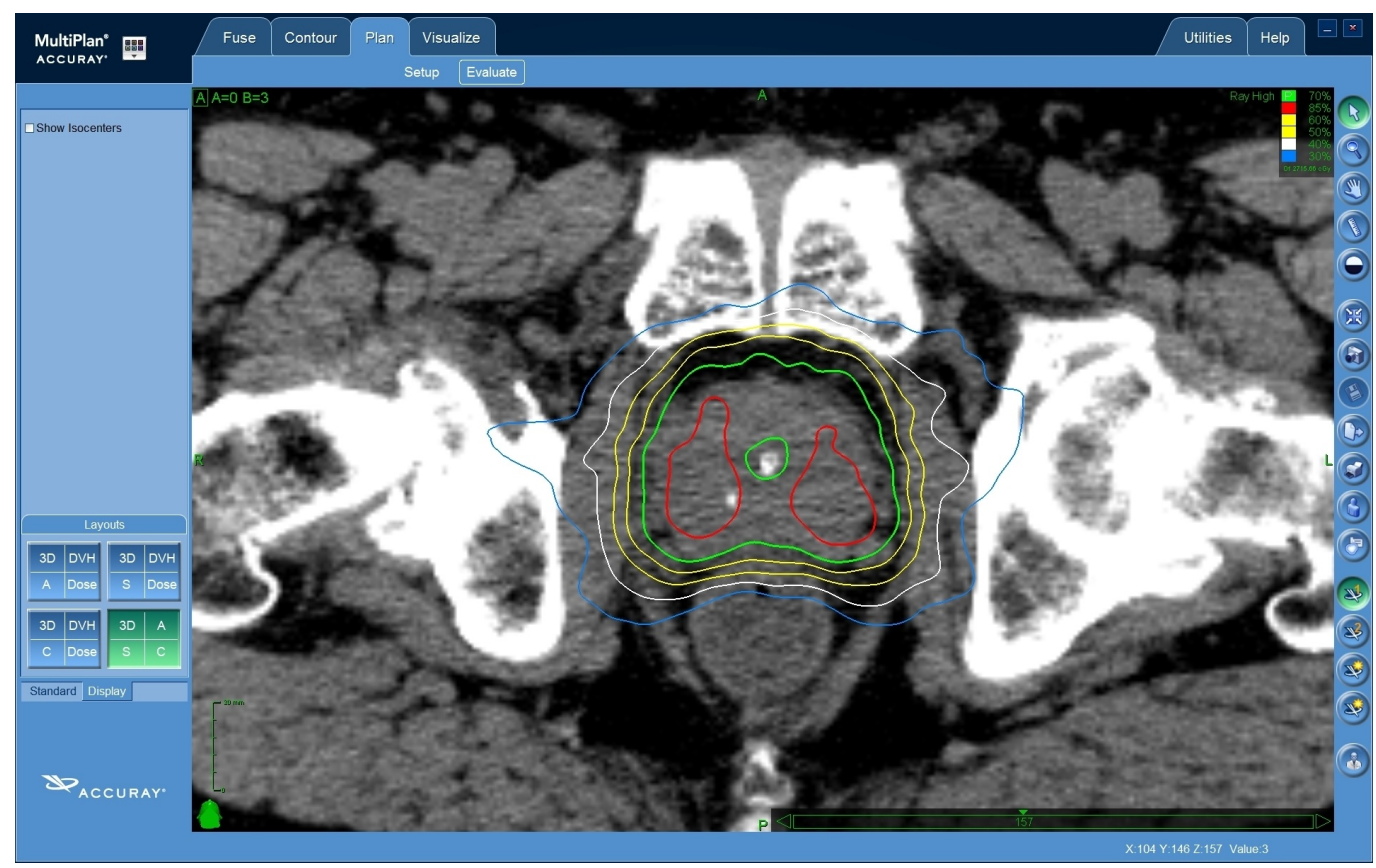

\section{FIGURE 2: Treatment planning}

Figure 2 shows the isodoses around the prostate. 19 Gy were prescribed to the $70 \%$ isodose (PTV, green line), $20 \%$ of the volume received $>23 \mathrm{~Gy}$ ( $85 \%$ isodose, red line). Of note is the steep gradient towards the rectum.

The treatment was executed as an outpatient procedure with a Foley catheter in place. The prostate was located using the implanted fiducials. Organ motion was compensated in six degrees of freedom. The treatment was followed closely by a specialized medical physicist to achieve best possible tracking results. Immediately after treatment, the Foley catheter was removed and the patient was discharged home. Dexamethasone, $4 \mathrm{mg}$ daily for one week, was prescribed.

\section{Follow-up evaluation}

PSA value controls and clinical follow up were performed at three-month intervals in the first year after stereotactic radiosurgery (SRS). Urinary symptoms and quality of life was evaluated every six months by the International Prostate Symptom Score (IPSS) and sexual function by the International Index of Erectile Function (IIEF).

\section{Treatment response, toxicity}

PSA response after completion of treatment showed a gradual decline from initially $5.05 \mathrm{ng} / \mathrm{dl}$ to $0.07 \mathrm{ng} / \mathrm{dl}$ at last follow-up. The single fraction treatment was very well tolerated, resuming normal activities on the next day after treatment. Acute symptoms, namely Grade I dysuria, urinary urgency, frequency, and nocturia, resolved completely in the third week after treatment completion. Initial IPSS and IIEF before treatment was $8+2$ and 16 respectively. At last followup, an IPSS of $11+1$ and IIEF of 13 were documented. There was no rectal or other toxicity in the follow-up period.

\section{Discussion}

Herein, we describe an unconventional approach using SRS for a patient with a low-risk prostate cancer. The rationale for treating this patient in one fraction is based on 
radiobiological considerations that suggest hypofractionated radiosurgical treatment may be advantageous for prostate cancer. Although there was no final conclusion, contemporary data suggest the $\alpha / \beta$ ratio for prostate cancer tissue may be as low as $1.5 \mathrm{~Gy}$ [6]. This means that in addition to causing effective cancer cellular ablation and tissue sparing due to its physics attributes, a course of CyberKnife prostate radiosurgery may also create a favorable therapeutic ratio by virtue of the radiobiologic sensitivity of prostate cancer itself [6]. Other groups are also starting to think in a similar direction. HDR pioneers from the Beaumont Hospital in Michigan just recently announced a new single session HDR trial [11]. Interestingly, they are using exactly the same dose for their HDR treatment as we used in our current study (19 Gy). The prescription dose of $19 \mathrm{~Gy}$ has been selected after extrapolating the $4 \mathrm{x} 9.5$ protocol [5] to an equivalent single dose, which calculates to $20 \mathrm{~Gy}$ in one fraction. Therefore, we believed this would be an adequate dose to start with while being aware there is no reliable calculation method available.

The feasibility of CyberKnife for treating localized prostate cancer was first described by King, et al. at Stanford University [1]. Their Phase I protocol delivered 36.25 Gy in five fractions of $7.25 \mathrm{~Gy}$ [1]. In a recent report of acute and 18-month late toxicity in 26 low-risk patients, no patient experienced Grade 3 or 4 acute or late toxicity, and only one patient experienced a Grade 2 late morbidity (urethral stricture). Mean PSA 18 months after treatment was 0.22 $\mathrm{ng} / \mathrm{ml}$. Just recently, the pooled data of 1,100 patients with clinically localized prostate cancer of prospective Phase 2 clinical trials of SBRT from eight institutions were published. SBRT, using the CyberKnife, delivered a median dose of $36.25 \mathrm{~Gy}$ in four to five fractions. Patients were low-risk (58\%), intermediate-risk (30\%), and high-risk (11\%). A short-course of androgen deprivation therapy (ADT) was given to 14\%. The five-year biochemical relapse free survival rate was $93 \%$ for all patients; $95 \%, 83 \%$, and $78 \%$ for GS $\leq 6,7$, and $\geq 8$, respectively ( $\mathrm{p}=0.001)$, and $95 \%, 84 \%$, and $81 \%$ for low-, intermediate- and high-risk patients, respectively $(p<0.001)$. For 135 patients possessing a minimum of five years follow-up, the five-year bRFS rate for low- and intermediate-risk patients was $99 \%$ and $93 \%$, respectively [12].

The main technical challenge of external beam radiation therapy for prostate treatment is that the prostate may move substantially, both between fractions (interfraction motion) and during the treatment itself (intrafraction motion), even if rigid body immobilization is applied to compensate for organ movement [6-7]. This prostate motion effect inherently requires the application of a larger radiotherapy planning target volume when tracking in all angles and directions (six-dimensional tracking) is not employed [13]. The unique tracking capabilities of the CyberKnife technology can compensate for prostate motion during treatment and therefore allow a focused external radiation treatment with potentially lower toxicity to the surrounding sensitive structures like the rectum and the bladder. However, with modern cone beam technology and the ability to track fiducials, Linacs may also be able to do SBRT for prostate cancer, and decrease treatment time with Rapid Arc or VMAT. Also, a rectal balloon can markedly diminish the intrafraction movement [14].

Taking into account the reduction in the number of sessions from 35 and more fractions to five and four fractions in the current trials a next step to only one fraction may be justifiable from a radiobiological standpoint. We currently plan to start a well-defined prospective multicenter Phase I/II study for patients with low risk prostate cancer to better understand the effects of the herein described approach which could become a revolution in radiation treatment of prostate cancer.

\section{Conclusions}

This is the first description of single session prostate radiosurgery for low-grade prostate cancer. The six-year follow up was uneventful, and the last PSA control was $0.07 \mathrm{ng} / \mathrm{dl}$. We suggest a prospective clinical trial on single session prostate radiosurgery to elucidate the 
options for this innovative treatment approach for low-grade prostate cancer.

\section{Additional Information \\ Disclosures}

Human subjects: Consent was obtained by all participants in this study. Conflicts of interest: In compliance with the ICMJE uniform disclosure form, all authors declare the following:

Payment/services info: All authors have declared that no financial support was received from any organization for the submitted work. Financial relationships: All authors have declared that they have no financial relationships at present or within the previous three years with any organizations that might have an interest in the submitted work. Other relationships: Alexander Muacevic, MD is operator of the Cyberknife Center Munich.

\section{References}

1. King CR, Lehmann J, Adler JR, Hai J: CyberKnife radiotherapy for localized prostate cancer: rationale and technical feasibility. Technol Cancer Res Treat. 2003, 2:25-30.

2. Hara W, Patel D, Pawlicki T, Cotrutz C, Presti J, King C: Hypofractionated Stereotactic Radiotherapy for Prostate Cancer: Early Results. Int J Radiat Oncol Biol Phys. 2006, 66:S324S325. 10.1016/j.ijrobp.2006.07.612

3. Freeman DE, King CR: Stereotactic body radiotherapy for low-risk prostate cancer: Five-year outcomes. Radiat Oncol. 2011, 6:3. 10.1186/1748-717X-6-3

4. Katz AJ, Santoro M, Ashley R, Diblasio F, Witten M: Stereotactic body radiotherapy for organconfined prostate cancer. BMC Urol. 2010, 10:1. 10.1186/1471-2490-10-1

5. Fuller DB, Naitoh J, Lee C, Hardy S, Jin H: Virtual HDR CyberKnife treatment for localized prostatic carcinoma: Dosimetry comparison with HDR brachytherapy and preliminary clinical observations. Int J Radiat Oncol Biol Phys. 2008, 70:1588-97. 10.1016/j.ijrobp.2007.11.067

6. Fung AY, Enke CA, Ayyangar KM, Raman NV, Zhen W, Thompson RB, Li S, Nehru RM, Pillai S: Prostate motion and isocenter adjustment from ultrasound-based localization during delivery of radiation therapy. Int J Radiat Oncol Biol Phys. 2005, 61:984-92.

7. Wong JR, Grimm L, Uematsu M, Oren R, Cheng CW, Merrick S, Schiff P: Image-guided radiotherapy for prostate cancer by CT-linear accelerator combination: Prostate movements and dosimetric considerations. Int J Radiat Oncol Biol Phys. 2005, 61:561-9.

8. Kupelian PA, Thakkar VV, Khuntia D, Reddy CA, Klein EA, Mahadevan A: Hypofractionated intensity-modulated radiotherapy ( 70 gy at $2.5 \mathrm{~Gy}$ per fraction) for localized prostate cancer: Long-term outcomes. Int J Radiat Oncol Biol Phys. 2005, 63:1463-8.

9. Chang SD, Main W, Martin DP, Gibbs IC, Heilbrun MP: An analysis of the accuracy of the CyberKnife: A robotic frameless stereotactic radiosurgical system. Neurosurg. 2003, 52:140-6.

10. Fowler JF: The radiobiology of prostate cancer including new aspects of fractionated radiotherapy. Acta Oncol. 2005, 44:265-76.

11. Beaumont Launches Study of Single-Dose Radiation Treatment for Prostate Cancer . (2014). Accessed: August 1, 2014: http://dr.beaumontphysician.com/news/pages/single-dosebrachytherapy-treatment.aspx.

12. King CR, Freeman D, Kaplan I, Fuller D, Bolzicco G, Collins S, Meier R, Wang J, Kupelian P, Steinberg M, Katz A: Stereotactic body radiotherapy for localized prostate cancer: pooled analysis from a multi-institutional consortium of prospective phase II trials. Radiother Oncol. 2013, 109:217-21. 10.1016/j.radonc.2013.08.030

13. van de Water S, Valli L, Aluwini S, Lanconelli N, Heijmen B, Hoogeman M: Intrafraction prostate translations and rotations during hypofractionated robotic radiation surgery: Dosimetric impact of correction strategies and margins. Int J Radiat Oncol Biol Phys. 2014, 88:1154-60. 10.1016/j.ijrobp.2013.12.045

14. Loblaw A, Cheung P, D'Alimonte L, Deabreu A, Mamedov A, Zhang L, Tang C, Quon H, Jain S, Pang G, Nam R: Prostate stereotactic ablative body radiotherapy using a standard linear accelerator: Toxicity, biochemical, and pathological outcomes. Radiother Oncol. 2013, 107:153-8. 10.1016/j.radonc.2013.03.022 